http://jmscr.igmpublication.org/home/ ISSN (e)-2347-176x ISSN (p) 2455-0450

crossref DOI: https://dx.doi.org/10.18535/jmscr/v8i3.82

Journal Of Medical Science And Clinical Research

\title{
Effects of Treatment on Pulmonary Function Tests in Patients of Chronic Rhinosinusitis
}

\author{
Authors \\ Dr Varun Rawat ${ }^{1}$, Dr Bhawana Pant ${ }^{* *}$, Dr Vaibhav Kuchhal ${ }^{3}$, Dr Ravi Baniya ${ }^{4}$ \\ Dr Shikha Bhatt ${ }^{5}$, Dr Aakanksha Rawat ${ }^{6}$ \\ ${ }^{1,4,5,6}$ PG Resident 3rd yr ENT Dept GMC Haldwani Uttrakhand \\ ${ }^{2}$ Associate Professor ENT Dept, Doon Medical College, Dehradun, Uttrakhand \\ ${ }^{3}$ Professor and Head of ENT Dept GMC Haldwani Uttrakhand \\ *Corresponding Author \\ Dr Bhawana Pant
}

\begin{abstract}
Background: Chronic Rhinosinusitis (CRS), is an inflammatory condition of the nose and paranasal sinuses persisting for at least 12 weeks characterized by 2 or more nasal/sinus symptoms, one of which includes nasal obstruction or nasal discharge, facial pressure/pain and reduced smell.

Methods: 60 Patients with features of Chronic Rhinosinusitis were examined in the ENT OPD. All participants underwent nasal endoscopic examination and CT scanning paranasally. Spirometry was performed to assess baseline pulmonary function. Subjects were divided into two treatment groups medical and medical plus surgical treatment group.

Results: It was inferred that the patients of chronic rhino-sinusitis had reduced baseline Pulmonary function test values. The effect of medical management on PFT was not found significant. Mean FVC and FEVI in MT group was found to higher post treatment as compared to pre-treatment but only the difference for FVC was found to be statistically significant. For FEV1/FVC the mean was higher prior to treatment as compared to post treatment however this difference was not found to be statistically significant.

The patients with combined treatment had significant improvement in PFT Mean FVC, FEV1 and FEVI/FVC was higher post treatment as compared to pre-treatment in MS treatment group and also this difference was found to be statistically significant.

Conclusions: It was found that patients with Chronic rhinosinusitis had decreased Pulmonary Function Test. The effect of medical management on PFT was not found significant. The patients with combined treatment had significant improvement in PFT.

Keywords: Chronic Rhinosinusitis, Pulmonary Function test, Spirometry.
\end{abstract}

\section{Definition}

Chronic Rhinosinusitis (CRS), is an inflammatory condition of the nose and paranasal sinuses persisting for at least 12 weeks characterized by 2 or more nasal/sinus symptoms, one of which includes nasal obstruction or nasal discharge, facial pressure/pain and reduced smell. In addition, endoscopic signs of mucopurulent discharge or edematous/mucosal obstruction of the middle meatus or radiologic abnormalities, 
such as mucosal changes within the ostiomeatal complex or sinuses, support the diagnosis. ${ }^{1}$ (Fokkens WJ)

\section{Prevalence}

Globally, the prevalence of CRS is variable with occurrence above $10 \%$ in Europe and the United States but lower at $2 \%$ among populations in sub Saharan Africa. ${ }^{2}$

CRS is one of the most commonly diagnosed diseases of the upper airways not only in western countries but also increasingly in Asia. ${ }^{3}$

When the onset of these symptoms is acute and when they are present for less than twelve weeks, the diagnosis is Acute Rhinosinusitis (ARS). When they persist for more than twelve weeks, the diagnosis is Chronic Rhinosinusitis (CRS) It has two subtypes Chronic Rhinosinusitis without nasal polyps (CRSsNP) and Chronic Rhinosinusitis with nasal polyps (CRSwNP). ${ }^{4}$

\section{Pulmonary function tests}

Pulmonary function tests (PFTS) are an important tool in the investigation and monitoring of patients with respiratory pathology. Although they do not provide a diagnosis per se, different patterns of abnormalities are seen in various respiratory diseases which helps to establish a diagnosis. ${ }^{5}$

\section{Spirometry}

Spirometry is the most frequently used measure of lung function and is a measure of volume against time. It is a simple and quick procedure to perform: patients are asked to take a maximal inspiration and then to forcefully expel air for as long and as quickly as possible (a forced vital capacity manoeuvre). Measurements that are made include

- Forced expiratory volume in one second (FEV1)

- Forced vital capacity (FVC)

- The ratio of the two volumes (FEV1/FVC)

The graphic display from spirometry is typically called a flow-volume curve if it includes only expiratory flow or a flow-volume loop if it includes both expiratory and inspiratory maneuvers (for simplicity, the rest of this review refers only to the flow-volume loop). The flowvolume loop provides important clues about the quality, acceptability, and reproducibility of the maneuver, which is determined by national standards and controlled by each individual laboratory. It can also indicate unusual abnormalities, such as obstructive lesions of the central airways. Inspiratory flows are disproportionately reduced by lesions of the upper (extrathoracic) airway. Conversely, lesions in the lower trachea and main stem bronchi primarily affect expiratory flows whereas a plateau on both the inspiratory and expiratory curves suggests a fixed lesion.The abnormal results of spirometry separate into 2 large classes of disorders: obstructive and restrictive. Obstructive disorders are suggested by a low FEV1/FVC ratio, whereas restrictive disorders are suggested by a normal FEV1/FVC ratio with a low FVC. ${ }^{6}$

\section{Medical management of Chronic Rhinosinusitis}

Daily use of Intranasal Corticosteroids (INCs) is the mainstay of therapy for CRS, especially for persons with nasal polyposis. INCSs serve to modify the disease process by decreasing the number of inflammatory cells (eosinophils, $\mathrm{T}$ lymphocytes, dendritic cells, and mast cells) through inhibition of their migration out of the circulation and survival in tissue. Oral corticosteroids are often used for exacerbations of disease because they result in improvement in both objective (polyp size) and subjective (hyposmia score) measures. The use of nasal saline irrigation is common in CRS, and data have indicated that it improves symptoms.

\section{Endoscopic Sinus Surgery on Patients with Chronic Rhinosinusitis}

Endocopic Sinus Surgery (ESS) is most frequently done for inflammatory and infectious sinus disease. The most common indications for ESS are as follows: 
i. CRS refractory to medical treatment,

ii. Recurrent sinusitis,

iii. Nasal polyposis,

iv. Antrochoanal polyps,

v. Sinus mucoceles,

vi. Excision of selected tumours,

Functional Endoscopic Sinus Surgery (FESS) is a type surgery of sinus conducted through the nose utilising endoscopes and inciting no external scars, is an innovative method in the management armamentarium of sinus disorder. 'FESS is a minimally invasive technique used to restore sinus ventilation and normal function. ${ }^{7}$

According to Yousuf et al ${ }^{8}$ it was found that pulmonary function tests in patients with chronic rhinosinusitis is significantly lower and the improvement in their sinus condition can lead to an improvement in their pulmonary function tests. The present study was done to evaluate baseline pulmonary functions test in patients with chronic rhinosinusitis (CRS) .Further evaluation was done to see the effects of medical treatment and combined (medical and surgical) management on pulmonary function tests of these patients.

\section{Materials and Methods}

\section{Patient selection}

The study was conducted in the department of Otorhinolaryngology, from January 2018 to September 2019. It was a prospective study. 60 patients in the age group of $18 \mathrm{yr}-55 \mathrm{yrs}$ of chronic rhinosinusitis with no lower airway compromise were selected for the study. Patients with previous facial trauma, acute rhinosinusitis, para nasal sinus malignancy, extra paranasal sinus tumor involving the sinuses. Patients with clinical evidence of sinusitis of dental origin, immunocompromised status. fungal rhinosinusitis, co-existent systemic disease like COPD, Bronchial asthma, diabetes, hypertension, neoplasia, prior paranasal sinus or throat surgery were excluded from the study.

\section{Patient Assessment}

Patients with complaints of facial pain, facial congestion, nasal obstruction and nasal discharge of 12 weeks were examined in the ENT OPD. All participants underwent nasal endoscopic examination and CT scanning paranasally. LundKennedy endoscopy and Lund-Mackay CT scores were calculated. Each patient was sent to medicine department for check up by physician and X-ray chest was done to exclude any lower airway disease.

Spirometry was performed to assess baseline pulmonary function using Spiro Excel, PC based Pulmonary Function Test, Medicaid Systems (ISO 13485: 2003 Company). 60 cases of chronic rhinosinusitis were selected and divided into two groups. Group 1 (medical treatment group (MT) were subjected to the medical management. Group 2 (medically resistant subjects (MS) who underwent combined (surgical and medical) management.

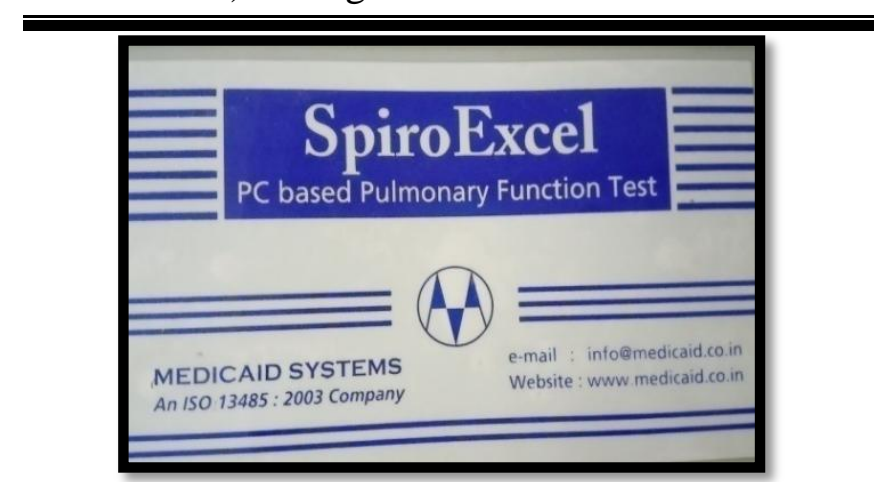

Fig. 1 Spirometer used for Pulmonary Function Test

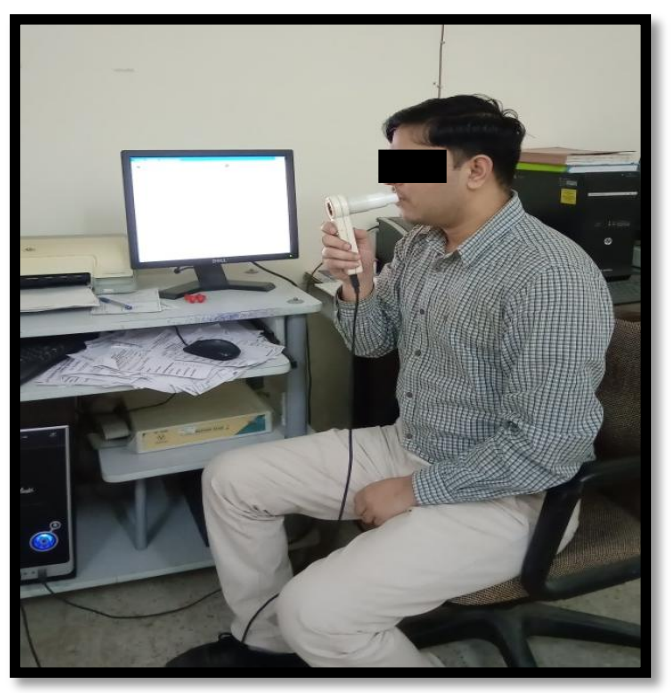

Fig. 2 Spirometry Procedure 


\section{Statistical Analysis}

Test of significance, correlation coefficient \& comparison of performance scores using fisher paired " $t$ "e test were calculated. Data was analysed using SPSS version 16.0 software. A p value of < 0.05 was considered statistically significant. All these findings were documented as per the study performa.

\section{Results}

The study comprised of 60 patients was conducted in the Department of Otorhinolaryngology and Department of Medicine from January 2018 to September 2019.

\section{Distribution of Cases}

1-Pre treatment Pulmonary Function Tests (PFT) MT Group-

The baseline pre-treatment mean FVC , FEV1 and FEV1/FVC in MT treatment group was $2.70 \pm 0.45,2.34 \pm 0.41$ and $87.67 \pm 3.98$ as shown in Fig.3.

Fig.3

\begin{tabular}{lc}
\hline Variable & Mean \pm SD \\
\hline FVC & $2.70 \pm 0.45$ \\
FEV1 & $2.34 \pm 0.41$ \\
FEV1/FVC & $87.67 \pm 3.98$ \\
\hline
\end{tabular}

\section{2-Post treatment Pulmonary Function Tests (PFT) of MT Group}

The baseline post treatment PFT of MT Group showed that the Post-treatment mean FVC, FEV1 and FEV1/FVC in MT treatment group was $2.88 \pm 0.45,2.41 \pm 0.52$ and $87.24 \pm 4.09$ respectively.

Fig.4

\begin{tabular}{lc}
\hline Variable & Mean \pm SD \\
\hline FVC & $2.88 \pm 0.45$ \\
FEV1 & $2.41 \pm 0.52$ \\
FEV1/FVC & $87.24 \pm 4.09$ \\
\hline
\end{tabular}

3-Pre treatment Pulmonary function Tests MT/MS Group

The Pre-treatment mean FVC, FEV1 and FEV1/FVC in MS treatment group was $2.42 \pm 0.79$, $2.27 \pm 0.75$ and $85.24 \pm 6.58$ respectively
Fig.5

\begin{tabular}{lc}
\hline Variable & Mean \pm SD \\
\hline FVC & $2.42 \pm 0.79$ \\
FEV1 & $2.27 \pm 0.75$ \\
FEV1/FVC & $85.24 \pm 6.58$ \\
\hline
\end{tabular}

4-Post treatment Pulmonary Function Tests MT/MS group

The Post-treatment mean FVC, FEV1 and FEV1/FVC in MS treatment group was 4.07 \pm 1.01 , $3.42 \pm 0.92$ and $86.37 \pm 4.97$ respectively

Fig.6

\begin{tabular}{lc}
\hline Variable & Mean \pm SD \\
\hline FVC & $4.07 \pm 1.01$ \\
FEV1 & $3.42 \pm 0.92$ \\
FEV1/FVC & $86.37 \pm 4.97$ \\
\hline
\end{tabular}

\section{5-Comparison between Pre and Post treatment PFT of MT Group (group 1)}

The mean FVC and FEV1 in MT treatment group was found to higher post treatment as compared to pre-treatment but only the difference for FVC was found to be statistically significant. For FEV1/FVC the mean was higher prior to treatment as compared to post treatment however this difference was not found to be statistically significant.

Fig 7

\begin{tabular}{lccc}
\hline Variable & $\begin{array}{c}\text { Pre- } \\
\text { Treatment }\end{array}$ & Post-Treatment & $\begin{array}{c}\mathbf{p} \\
\text { Value* }\end{array}$ \\
\cline { 2 - 3 } & Mean \pm SD & Mean \pm SD & \\
FVC & $2.70 \pm 0.45$ & $2.88 \pm 0.45$ & 0.004 \\
FEV1 & $2.34 \pm 0.41$ & $2.41 \pm 0.52$ & 0.23 \\
FEV1/FVC & $87.67 \pm 3.98$ & $87.24 \pm 4.09$ & 0.06 \\
\hline
\end{tabular}

*Paired sample t Test

6.Comparison between Pre and Post treatment PFT of Combined (medical and surgical) Group 2

The mean FVC, FEV1 and FEV1/FVC was higher post treatment as compared to pre-treatment in MS treatment group and also this difference was found to be statistically significant.

Fig.8

\begin{tabular}{|l|c|c|c|}
\hline Variable & $\begin{array}{c}\text { Pre- } \\
\text { Treatment }\end{array}$ & $\begin{array}{c}\text { Post- } \\
\text { Treatment }\end{array}$ & \multirow{2}{*}{ p Value* } \\
\cline { 2 - 3 } & Mean \pm SD & Mean \pm SD & \\
\hline FVC & $2.42 \pm 0.79$ & $4.07 \pm 1.01$ & 0.001 \\
\hline FEV1 & $2.27 \pm 0.75$ & $3.42 \pm 0.92$ & 0.001 \\
\hline FEV1/FVC & $85.24 \pm 6.58$ & $86.37 \pm 4.97$ & 0.001 \\
\hline
\end{tabular}




\section{Discussion}

Treatment of chronic rhino-sinusitis is primarily medical with glucocorticoids have formed the mainstay of medical therapy for CRS, with surgery reserved for those who fail a trial of maximal medical treatment(Usually 4-8 weeks should be allowed before considering treatment to have failed in patients with established CRS). The concept of 'one airway, one disease' and the 'united airway' in which the upper and lower airways act as a whole unit has been proposed, with rhinitis and asthma representing manifestations of the same inflammatory process ${ }^{8}$ since pulmonary function tests (PFTS) act as an important tool in the investigation and monitoring of patients with respiratory pathology we used spirometry values to assess the overall improvement in lower respiratory tract while we provide medical management as compare to surgical management.

This study was conducted to compare the Pulmonary Function Tests (PFT) of patients diagnosed with Chronic Rhinosinusitis. Patients were divided into two groups undergoing medical management and surgical followed by medical management. Pulmonary Function Tests were performed and values were compared pre and post treatment. The study included patients of varied age groups, varied height weight and both sexes. The results were compared with the available literature.Total 60 cases of chronic rhinosinusitis were taken, 20 cases in medical treatment group and 40 cases in combined treatment (medical and surgical) group.

In our study, it was found that Pulmonary Function Tests values were reduced in subjects with Chronic Rhinosinusitis having no active pulmonary disease which is in agreement with the study done by Tanaka et al. ${ }^{11}$ It was found that patients with chronic rhinosinusitis had decreased pulmonary function test with asymptomatic obstructive pattern observed in spirometry. The findings were due to bronchial hyperresponsiveness, altered ciliary motility of nose and paranasal sinus mucosa. It was also observed that management of these patients improved their pulmonary function. It was found that patients with Chronic Rhinosinusitis had non symptomatic lower airway involvement mainly terminal and respiratory bronchioles (small airway disease) which was assessed through spirometry but in medically treated patients there was no significant improvement in pulmonary function tests as suggested by Fig.7.

The pulmonary function tests values were reduced in medically treated subjects but to a lesser extent due to reduced severity of the disease according to diagnostic and radiological findings. Therefore, there was no significant improvement after medical treatment. ${ }^{12}$ Medically resistant patients had greater severity of disease and were not benefitted with the medical treatment. The pulmonary function tests values were significantly reduced due to excessive narrowing of airway, bronchial hyperreactivity and nasal obstruction ${ }^{10}$. These patients were subjected for combined (medical and surgical) treatment and it was observed that there was significant improvement in pulmonary function suggested by Fig.8.This improvement after surgery followed by medical treatment was the result of removal of triggering areas in nose and sinuses that causes release of leukotrienes, prostaglandins and other inflammatory mediators that may affect lower airway $^{8}$. It was revealed that a reduction of corticosteroid medication following ESS, but did not use any objective pulmonary function tests. The effect of postoperative topical application of corticosteroids to the nasal cavity in asthma remains undetermined. However, no clinical efficacy was obtained in control patients receiving intranasal corticosteroid spray alone, and this finding suggests that the spray made little contribution to sinus or bronchial disease. Thus, the present study supports that medicine plus surgery has a beneficial effect in associated with chronic sinusitis on the basis of objective pulmonary function tests as compare to medicinal treatment only. Kariya et $\mathrm{al}^{12}$ also reported that 
pulmonary functions were affected in patients with CRS regardless of their sensitization status.

$=$ The peak expiratory flow correlates well with forced expiratory volume in 1 second, one of the accepted parameters for evaluating the severity of obstructive conditions of the airway. ${ }^{9}$ In contrast, several investigators have mentioned that the peak expiratory flow is not really an adequate measure of lung function per se. However, because of seasonal and daily variation of the airway caliber in asthmatic patients, the average peak expiratory flow for only 1 month, in the same month, preoperatively and postoperatively, rather than spirometric parameters, was adopted as a practical and convenient pulmonary function test in the present study.

\section{Pulmonary Function Tests}

In the present study, it was noted that pretreatment mean FVC, FEV1 and FEV1/ FVC was higher for Medical treatment group, MT $(2.70 \pm 0.45,2.34 \pm 0.41,87.67 \pm 3.98)$ as compared to combined (Medical and surgical), MS treatment group $(2.42 \pm 0.79,2.27 \pm 0.75,85.24 \pm 6.58)$ and these differences were found to be statistically significant(p value $0.008,0.001,0.03$ ). In MS Group the pre-treatment mean FVC, FEV1 and FEV1/FVC was $2.42 \pm 0.79, \quad 2.27 \pm 0.75$ and $85.24 \pm 6.58$ respectively while post-treatment mean FVC, FEV1 and FEV1/FVC in MS treatment group was $4.07 \pm 1.01,3.42 \pm 0.92$ and $86.37 \pm 4.97$ respectively. This improvement can be attributed mainly to the surgery with steroid sprays and antibiotics also playing a role.

The present study revealed a significant improvement in pulmonary function of patients associated with chronic sinusitis following medicinal plus surgical treatment.

Although our study is limited by a relatively small number of patients, we believe that this prospective study, with its well-defined outcome measures and criteria included for patient selection, helps to clarify the true value of ESS for these difficult-to-treat patients and to emphasize that the underuse of objective testing, such as spirometry, for patients with CRS may lead to under diagnosed lower airway problems. Early diagnosis and good CRS control are important to reduce morbidity and health care costs as well as to minimize the development of chronic illnesses.

\section{Conclusion}

The following conclusions were drawn from the present study-

1) The patients of chronic rhino-sinusitis had reduced baseline Pulmonary function test values.

2) The effect of medical management on PFT was not found significant. Mean FVC and FEV1 in MT group was found to higher post treatment as compared to pre-treatment but only the difference for FVC was found to be statistically significant. For FEV1/FVC the mean was higher prior to treatment as compared to post treatment however this difference was not found to be statistically significant.

3) The patients with combined treatment had significant improvement in PFT Mean FVC, FEV1 and FEV1/FVC was higher post treatment as compared to pre-treatment in MS treatment group and also this difference was found to be statistically significant.

4) Post treatment mean FVC and FEV1 was higher for MS treatment group as compared to MT treatment group and this difference was found to be statistically significant. Mean FEV1/FVC was found to higher for MT treatment group as compared to MS treatment group however this difference was not found to be statistically significant.

\section{References}

1. Seong H. Cho et al. Chronic Rhinosinusitis Phenotypes: An Approach to Better Medical Care for Chronic Rhinosinusitis. J Allergy Clin Immunol Pract. 2016 ; 4(4): 639-642.

2. Victoria Nyaiteera. The burden of chronic rhinosinusitis and its effect on quality of life among patients re-attending an 
otolaryngology clinic in south western

Uganda. BMC Ear, Nose and Throat Disorders (2018) 18:10.

3. Yuan Zhang, Chronic rhinosinusitis in Asia. (J Allergy Clin Immunol 2017;140: 1230-9.)

4. Christopher Ocampo CJ, Grammer LC. Grand rounds: Chronic rhinosinusitis. J Allergy Clin Immunol: In Practice 2013;1:205-11.

5. Benninger et al. Adult chronic rhinosinusitis: Definitions, diagnosis, epidemiology, and pathophysiology. Otolaryngology-Head and Neck Surgery September 2003. Volume 129 number 3.

6. Harpreet Ranu, Pulmonary Function Tests. Ulster Med J 2011;80(2):84-90.

7. Milind V Kirtane.Textbook of endoscopic Endosnasal surgery, Sinuses and Beyond.

8. Ahmad M. Yousof. Pulmonary function tests in patients with chronic rhinosinusitis and the effect of surgery. Egyptian Journal of Bronchology, Vol. 11 No. 3, July-September 2018

9. Vaughan TR, Weber CRW, Tipton WR, Nelson HS. Comparison of PEFR and FEVi in patients with varying degrees of airway obstruction. Effect of modest altitude. Chest 1989;95: 558-62

10. Rodney P. Lusk Endoscopic sinus surgery in children with chronic sinusitis: A pilot study. The laryngoscope, 1990,vol 100, issue 6.

11. Tanaka S, Hirota T, Kamijo A, et al. Lung functions of Japanese patients with chronic rhinosinusitis who underwent endoscopic sinus surgery. Allergol Int. 2014;63:27-35.

12. Shin Kariya. Relationship between chronic rhinosinusitis and lower airway diseases: An extensive review. World $\mathbf{J}$ Otorhinolaryngol 2015 May 28; 5(2): 4452. (6). 